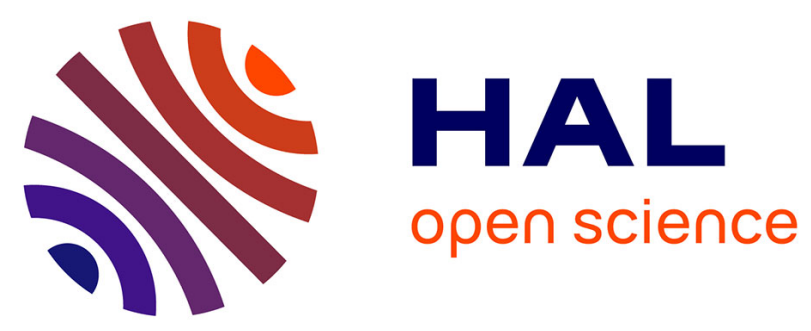

\title{
Perturbation de l'iode moléculaire en phase vapeur par la présence de gaz étrangers. Conséquence pour la stabilisation du laser He-Ne
}

G. Flory, M. Broyer, J. Vigue, J.C. Lehmann

\section{To cite this version:}

G. Flory, M. Broyer, J. Vigue, J.C. Lehmann. Perturbation de l'iode moléculaire en phase vapeur par la présence de gaz étrangers. Conséquence pour la stabilisation du laser He-Ne. Revue de Physique Appliquée, 1977, 12 (5), pp.901-904. 10.1051/rphysap:01977001205090100 . jpa-00244257

\section{HAL Id: jpa-00244257 https://hal.science/jpa-00244257}

Submitted on 1 Jan 1977

HAL is a multi-disciplinary open access archive for the deposit and dissemination of scientific research documents, whether they are published or not. The documents may come from teaching and research institutions in France or abroad, or from public or private research centers.
L'archive ouverte pluridisciplinaire HAL, est destinée au dépôt et à la diffusion de documents scientifiques de niveau recherche, publiés ou non, émanant des établissements d'enseignement et de recherche français ou étrangers, des laboratoires publics ou privés. 


\author{
Classification \\ Physics Abstracts \\ $5.446-5.448-0.460$
}

\title{
PERTURBATION DE L'IODE MOLÉCULAIRE EN PHASE VAPEUR PAR LA PRÉSENCE DE. GAZ ÉTRANGERS CONSÉQUENCE POUR LA STABILISATION DU LASER He-Ne
}

\author{
G. FLORY, M. BROYER, J. VIGUE et J. C. LEHMANN \\ Laboratoire de Spectroscopie Hertzienne de l'Ecole Normale Supérieure, \\ Université Pierre et Marie Curie, 4, place Jussieu, Tour 12, $1^{\text {er }}$ étage, 75230 Paris Cedex 05, France
}

(Reçu le 28 décembre 1976, accepté le 8 février 1977)

\begin{abstract}
Résumé. - Une méthode très sensible de mise en évidence des impuretés gazeuses dans les cellules d'iode moléculaire est présentée et appliquée. Les résultats prouvent la grande importance de ces impuretés. Nous montrons que la présence de ces gaz peut limiter la reproductibilité de la stabilisation sur l'iode du laser $\mathrm{He}-\mathrm{Ne}$. Une technique pour obtenir des cellules très propres est décrite et la qualité des cellules obtenues ainsi est analysée.
\end{abstract}

\begin{abstract}
A very sensitive method to test the presence of gaseous impurities in molecular iodine cells is presented and applied. The results prove the great importance of these impurities. It is shown that the presence of these gases can limit the reproducibility of the He-Ne laser stabilized on iodine. A technique to fill very clean cells is described and the quality of such cells is discussed.
\end{abstract}

1. Introduction. - Depuis plusieurs années, de nombreuses équipes [1] ont développé et amélioré le procédé de stabilisation en fréquence de la raie $6328 \AA$ d'un laser $\mathrm{He}-\mathrm{Ne}$ monomode sur l'un des pics d'absorption saturée de la vapeur d'iode moléculaire. Un étalon secondaire de longueur en est déjà résulté dont la reproductibilité avoisine $10^{-11}$.

Nous voulons attirer l'attention dans cette publication sur des limitations possibles de cet étalon dues à des effets de collisions sur des impuretés gazeuses, très fréquemment présentes dans les cellules scellées remplies d'iode.

2. Mise en évidence de gaz étrangers dans les cellules d'iode. - La mesure de la durée de vie $\tau$ d'un niveau moléculaire excité constitue un test de la présence de gaz étrangers. En effet, l'inverse $\gamma$ de cette durée de vie peut s'écrire :

$\gamma=\frac{1}{\tau}=\frac{1}{\tau_{0}}+\frac{V_{r I_{2} I_{2}^{*}}}{k T} \sigma_{I_{2} I_{2}^{*}}^{\mathrm{E}} p_{I_{2}}\left(T_{\mathrm{f}}\right)+\sum_{A} \frac{V_{r A I_{2}^{*}}^{*}}{k T} \sigma_{A I_{2}^{*}}^{\mathrm{E}} p_{A}\left(T_{\mathrm{f}}\right)$

$\tau_{0}$ désigne la durée de vie en l'absence totale de collisions $; I_{2}^{*}$ désigne l'iode excité et $v_{\mathrm{r} X Y}$ la vitesse relative moyenne des espèces $X$ et $Y$;

$\sigma^{\mathrm{E}}$ est la section efficace de destruction de la population du niveau excité de $I_{2}$ due aux collisions $I_{2} I_{2}^{*}$ ou $A I_{2}^{*}$.

Enfin, $T_{\mathrm{f}}$ est la température du point froid de la cellule et $p_{A}\left(T_{\mathrm{f}}\right)$ la pression de l'espèce atomique ou moléculaire $A$ présente dans la cellule ; on a forcément $p_{A}\left(T_{\mathrm{f}}\right) \leqslant p_{A}^{\mathbf{s}}\left(T_{\mathrm{f}}\right)$ où $p_{A}^{\mathbf{s}}\left(T_{\mathrm{f}}\right)$ désigne la pression de vapeur saturante de l'espèce $A$ à la température $T_{\mathrm{f}}$. L'iode est supposée toujours en vapeur saturante. $T$ est la température de la cellule, constante et toujours supérieure à $T_{\mathrm{f}} ; k$ est la constante de Boltzmann.

Le rapport signal sur bruit de toute mesure de $\gamma$ tend vers zéro lorsque $p_{I_{2}}$ tend vers zéro; l'étude de $\gamma$ ne peut se faire que pour $p_{I_{2}}>p_{0}$ soit $T_{\mathrm{f}}>T_{0}$. La zone $T_{\mathrm{f}}<T_{0}$ est inaccessible pratiquement (En général, pour nos mesures, l'ordre de grandeur de $p_{0}$ est de 2,5 mtorr ce qui correspond à $\left.T_{0}=-22^{\circ} \mathrm{C}\right)$. La formule utilisée pour relier $p_{I_{2}}$ à $T_{\mathrm{f}}$ est celle de Baxter et Grose [2]

$$
\left(\log p_{\mathbf{I}_{2}} \text { (torr) }=9,7522-\frac{2863,54}{273+t-19} ; t \text { en }{ }^{\circ} \mathrm{C}\right) \text {. }
$$

Si la cellule ne contient que de l'iode, $\gamma$ est une fonction linéaire de $p_{I_{2}}$ et l'extrapolation linéaire de $\gamma$ en fonction de $p_{I_{2}}$ donne $\gamma_{0}=1 / \tau_{0}$. C'est le cas idéal représenté par la courbe 1 sur la figure 1 .

Si la cellule contient une seule impureté $A$, en quantité faible, il existera une température du point froid $T_{A}$ en dessus de laquelle l'impureté $A$ sera en vapeur sèche de pression fixe $p_{A}=p_{A}^{\mathrm{s}}\left(T_{A}\right)$; pour $T_{\mathrm{f}}$ inférieur à $T_{A}$ nous admettrons que $p_{A}\left(T_{\mathrm{f}}\right)=p_{A} \mathbf{s}\left(T_{\mathrm{f}}\right)$; ceci est une bonne approximation s'il ne se produit pas de réaction chimique entre l'iode solide et la phase solide 


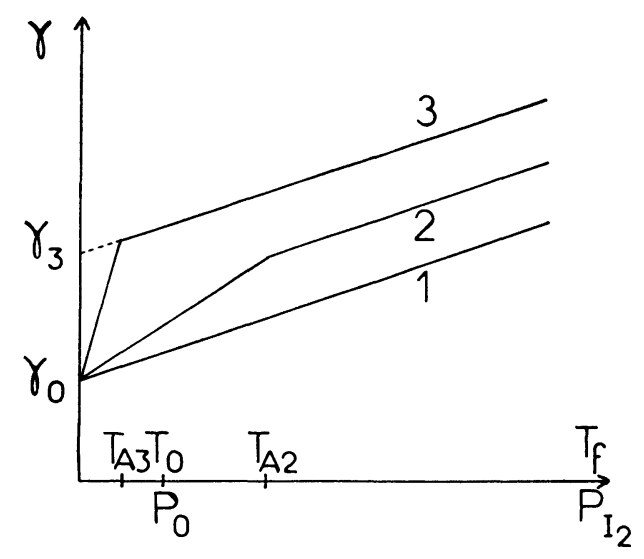

Fig. 1. - La dépendance théorique de $\gamma$ en fonction de la pression d'iode est représentée dans 3 cas. (L'échelle des abscisses est linéaire pour $\boldsymbol{P}_{\boldsymbol{I}_{2}}$ et non linéaire en $\boldsymbol{T}_{\mathrm{f}}$ ) Courbe 1 : cellule sans gaz étrangers. Courbes 2 et 3 : cellule avec gaz étrangers en vapeur sèche pour $T_{\mathrm{f}}>T_{A_{2}}$ et $T_{A_{3}}$ respectivement.

[The theoretical dependence of $\gamma$ as a function of the iodine pressure is plotted in 3 cases. (The horizontal scale is linear in $P_{I_{2}}$ and non linear in $T_{\mathrm{f}}$ ): Curve $1:$ cell free from foreign gases ; Curves 2 and $3:$ cell with foreign gases in unsaturated vapour for $T_{\mathrm{P}}>T_{A_{2}}$ and $T_{A_{3}}$ respectively.]

ou liquide de $A . \gamma$ en fonction de $p_{I_{2}}$ est alors représenté par une droite pour $T>T_{A}$, alors qu'au-dessous de $T_{A}$, la forme de $\gamma$ n'est pas simple, sauf si $p_{A}\left(T_{\mathrm{f}}\right)$ et $p_{I_{2}}\left(T_{\mathrm{f}}\right)$ sont proportionnels, auquel cas $\gamma$ y est encore représenté par une droite. Les courbes 2 et 3 de la figure 1 représentent ce cas avec soit $T_{A_{2}}>T_{0}$ soit $T_{A_{3}}<T_{0}$.

Les courbes 1 et 2 conduisent à la même valeur extrapolée de $\gamma$ mais avec des pentes différentes dans la zone $T_{\mathrm{f}}<T_{A_{2}}$. La courbe 3 s'extrapole à

$$
\gamma_{3}=\gamma_{0}+\frac{V_{r A I_{2}^{*}}}{k T} p_{A} \sigma_{A I_{2}^{*}}^{\mathrm{E}}
$$

car la zone $T<T_{0}$ n'est pas explorée. On voit donc que la comparaison des valeurs extrapolées de $\gamma$ obtenues pour des cellules différentes sera sensible à la présence d'impuretés telles que $T_{A}<T_{0}$. Si au contraire $T_{A}>T_{0}$, ce ne sera que la pente $\delta \gamma / \delta p_{I_{2}}$ qui sera sensible à la présence de l'impureté $A$.

Nous disposons donc d'une méthode de mise en évidence des impuretés. Cette méthode sera d'autant plus sensible que le terme $\gamma_{0}$ sera plus petit et que les sections efficaces seront grandes. L'iode est un cas très favorable dans la mesure où lorsqu'on s'approche de la limite de dissociation de l'état $\mathrm{B}^{3} \Pi_{\mathrm{ou}}^{+}$on trouve des niveaux d'énergie de durée de vie très longue [3] ; simultanément ces niveaux sont un peu plus sensibles aux collisions.

Le tableau I compare les durées de vie et les sections efficaces $I_{2}-I_{2}^{*}$ pour les niveaux $B^{3} \Pi_{\mathrm{ou}}^{+} v^{\prime}=11$ $J^{\prime}=128$ (niveau supérieur de la transition servant à l'asservissement du laser He-Ne $6328 \AA$ ) et du niveau $v^{\prime}=62 J^{\prime}=27$ excité par la raie $5017 \AA$ du laser $\mathrm{Ar}^{+}$.
Niveaux $B^{3} \Pi_{\mathrm{ou}}^{+}$:

$$
v^{\prime}=11 J^{\prime}=128 \text { [3] } v^{\prime}=62 J^{\prime}=27[4.5] \text {. }
$$

Distance à la limite de dissociation

$$
\begin{array}{lrr} 
& \multicolumn{1}{c}{3041 \mathrm{~cm}^{-1}} & \multicolumn{1}{c}{98 \mathrm{~cm}^{-1}} \\
\tau_{0}(\mu \mathrm{s}) & 0.410 \pm 0.015 & 14 \pm 3 \\
\sigma_{I_{2} I_{2}^{*}}^{\mathrm{E}}\left(\AA^{2}\right) & 204 \pm \quad 15 & 286 \pm 40
\end{array}
$$

3. Méthodes de mesure des durées de vie. Résultats. - Les deux méthodes que nous avons utilisées sont l'effet Hanle c'est-à-dire la dépolarisation magnétique de la fluorescence [4] et l'observation de la décroissance de la fluorescence suivant une excitation en impulsion [5]. Les résultats sont analogues. Nous pensons cependant que dans le cas du niveau $v^{\prime}=62 J^{\prime}=27$, l'effet Hanle peut s'avérer plus pratique car il ne nécessite pas d'électronique rapide mais seulement un champ magnétique assez faible balayable de 0 à $200 \mathrm{G}$ environ.

L'observation de l'effet Hanle donne des courbes de Lorentz d'absorption ou de dispersion en fonction du champ magnétique $B$, d'équations

$$
\frac{1}{1+\left(B / B_{0}\right)^{2}} \text { ou } \frac{\left(B / B_{0}\right)}{1+\left(B / B_{0}\right)^{2}} \text {. }
$$

La valeur de $B_{0}$ est reliée à $\gamma$ par

$$
2 g \mu_{\mathrm{n}} B_{0}=h \gamma
$$

soit d'après nos mesures du facteur de Landé $g[4,6]$

$$
B_{0}(\mathrm{G})=(5,7 \pm 0,2) 10^{-5} \gamma\left(\mathrm{s}^{-1}\right)
$$

équation valable seulement pour le niveau $v^{\prime}=62$ $J^{\prime}=27$.

La figure 2 montre l'inverse $\gamma$ de la durée de vie de ce niveau en fonction de la pression d'iode pour deux cellules différentes remplies dans des conditions analogues avec de l'iode ${ }^{127} I_{2}$, de haute pureté, après un étuvage de la cellule à $350^{\circ} \mathrm{C}$ pendant 24 heures sous

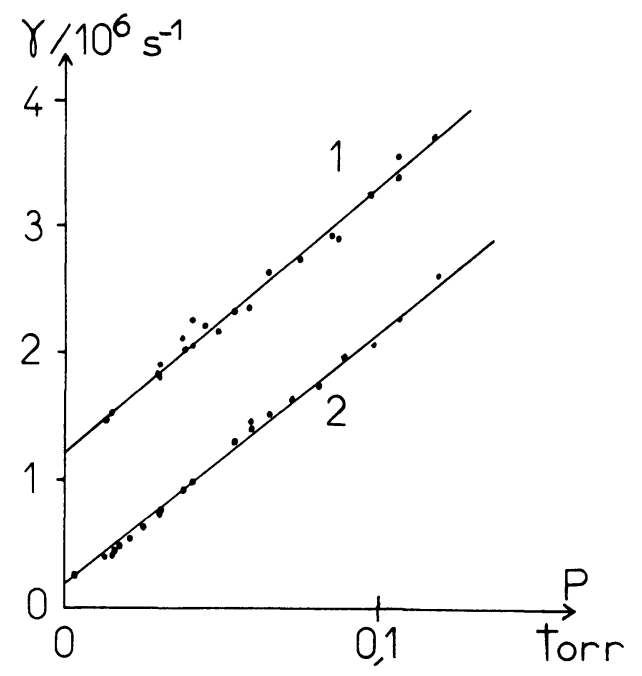

FIG. 2. - Dépendance expérimentale de $\gamma\left(v^{\prime}=62, J^{\prime}=27\right)$ pour deux cellules remplies dans des conditions différentes.

[Experimental dependence of $\gamma\left(v^{\prime}=62, J^{\prime}=27\right)$ for two cells filled in different conditions.] 
un vide meilleur $10^{-6} \mathrm{t}$. L'iode est introduit par distillation sous vide. Le piégeage a été effectué avec de l'azote liquide $(77 \mathrm{~K})$ pour la cellule 1 , avec un mélange alcool-carboglace $(195 \mathrm{~K})$ pour la cellule 2.

On voit que les pentes des deux droites sont les mêmes mais que les extrapolations donnent des résultats différents. C'est un cas typique où des impuretés sont présentes dans au moins une des deux cellules, ces impuretés ne se condensant pas au-dessus de $T=-22^{\circ} \mathrm{C}$. La différence des valeurs extrapolées de $\gamma$ atteint $10^{6} \mathrm{~s}^{-1}$.

Ces résultats proviennent de cellules récemment remplies. On constate que les résultats se dégradent avec le temps, c'est-à-dire que $\gamma$, extrapolé à pression d'iode nulle, croît.

4. Conséquences de ces résultats pour un étalon de longueur. - 4.1 LARGEUR ET DÉPLACEMENT D'UNE RAIE OPTIQUE DUS AUX COLLISIONS. - On sait que les collisions élargissent et déplacent une transition atomique. Aux faibles pressions ( $\sim 1$ torr) ces effets sont très inférieurs à la largeur Döppler, mais tout à fait observables en absorption saturée. Dans le cas de collisions iode-iode, pour la transition $\mathrm{B}^{3} \Pi_{\mathrm{ou}}^{+} \leftarrow X^{1} \Sigma_{\mathrm{g}}^{+}$ $11-5 \mathrm{R}(127)$ on trouve que $[7,8]$

$$
\begin{aligned}
\frac{\delta v}{\delta p_{I_{2}}} & =-1,12 \mathrm{MHz} / \text { torr } \\
\frac{\delta \Delta F}{\delta p_{I_{2}}} & =8,7 \mathrm{MHz} / \text { torr }
\end{aligned}
$$

$v$ étant la fréquence et $\Delta F$ la largeur de la transition.

Il n'y a pas d'autres mesures de ces quantités pour l'iode. Mais de nombreuses études de déplacementélargissement ont été faites sur les transitions électroniques atomiques. Il n'existe pas de relation générale entre $\delta \Delta F / \delta p$ et $\delta v / \delta p$; ni avec $\delta \gamma / \delta p$ (où $\gamma$ est l'inverse de la durée de vie de l'état excité de la transition).

4.2 EFFet SUR L'ÉTALON DE LONGUEUR D'UNE IMPURETÉ DANS LA CELlule. - Pour estimer les effets d'une impureté, nous allons admettre que

$$
\left(\frac{\delta v}{\delta p_{A}}\right) /\left(\frac{\delta v}{\delta p_{I_{2}}}\right) \simeq \frac{\sigma_{A I_{2}^{*}}^{\mathrm{E}} v_{r A I_{2}^{*}}}{\sigma_{I_{2} I_{2}^{*}}^{\mathrm{E}} v_{r_{2} I_{2}^{*}}}
$$

Cette hypothèse revient à admettre que l'impureté $A$ est équivalente à une certaine pression d'iode $p_{\text {eq, }}$, la même pour la destruction de la population de l'état excité que pour le déplacement de la raie optique. Cette hypothèse nous semble raisonnable pour obtenir un ordre de grandeur. La valeur de $p_{\text {eq }}$ se déduit aisément de la lecture de la figure 2 . On trouve $p_{\text {eq }} \simeq 5 \times 10^{-2}$ torr et on en déduit alors le déplacement dû à l'impureté présente dans la cellule 1 (Fig. 2) :

$$
\begin{aligned}
& \delta v=\left(\frac{\delta v}{\delta p_{A}}\right) p_{A} \simeq\left(\frac{\delta v}{\delta p_{I_{2}}}\right) p_{\text {eq }} \\
& \delta v \simeq 55 \mathrm{kHz}, \quad \frac{\delta v}{v}=1,2 \times 10^{-10} .
\end{aligned}
$$

Ce résultat est un ordre de grandeur du déplacement à craindre pour la raie. Une telle valeur constituerait une limitation essentielle de l'étalon $\mathrm{He} \mathrm{Ne}$-iode. Evidemment il est toujours possible que $\delta v / \delta p_{A}$ soit très petit. Une étude des déplacements de la raie avec la pression de corps très communs $\left(\mathrm{H}_{2} \mathrm{O}, \mathrm{CO}_{2}, \mathrm{O}_{2}, \mathrm{~N}_{2}\right)$ permettrait d'éclaircir ce point. Une façon probablement plus efficace d'aborder le problème consiste à réaliser des cellules très pures.

5. Méthode de fabrication de cellules très pures. Vieillissement de ces cellules. - Nous avons été amenés à fabriquer des cellules pratiquement exemptes d'impuretés gazeuses afin de pouvoir étudier les niveaux de longue durée de vie du type $\mathrm{B}^{3} \Pi_{\mathrm{ou}}^{+} v^{\prime}=62 \mathrm{~J}^{\prime}=27$.

Nous avons réussi à obtenir de telles cellules par une méthode que nous exposons ci-dessous. Cette méthode, basée sur quelques idées simples, n'est probablement pas la seule. Nous pourrons cependant en affirmer la qualité grâce aux tests très sensibles que sont les mesures de durée de vie du niveau $v^{\prime}=62$, $J^{\prime}=27$.

\subsection{RÉAlisAtion Des CELlUles. - Il faut résoudre} deux problèmes :

1) bonne qualité initiale des cellules ;

2) longue conservation de cette qualité.

Le vieillissement rapide des cellules remplies par la méthode évoquée plus haut est probablement dû à une réaction chimique de l'iode avec la cellule ellemême ou avec les impuretés absorbées dans les parois.

La cellule doit donc tout d'abord être étuvée sous vide $\left(p<10^{-6}\right.$ torr) à $350^{\circ} \mathrm{C}$ pendant $24 \mathrm{~h}$. On la remplit alors d'iode par distillation sous vide et la cellule scellée est à nouveau étuvée à $150^{\circ} \mathrm{C}$; la pression d'iode qui y règne alors est d'environ 400 torr, pression de vapeur saturante de l'iode à cette température. Le but de cette opération est de laver les parois, provoquant ainsi par avance les réactions qui pourraient avoir lieu. La cellule est alors remise en relation avec le banc de pompage sans rentrée d'air (par le bris d'une queue de cochon), vidée et réétuvée à $350^{\circ} \mathrm{C}$ pendant 24 heures. Cette première partie du traitement permet d'assurer une longue conservation à la cellule. Les valeurs de température sont adaptées aux cellules en pyrex. Elles devraient être modifiées pour des cellules en silice fondue.

Afin d'assurer la propreté initiale, l'iode utilisée pour le remplissage définitif est de haute pureté (par exemple Merck Suprapur); il subit d'abord une série de distillations aller et retour entre deux pièges que l'on refroidit alternativement à $-60^{\circ} \mathrm{C}$ environ. Ceci permet d'éliminer la vapeur d'eau qui est probablement le polluant le plus abondant et le plus volatif de l'iode (les gaz $\mathrm{N}_{2}, \mathrm{O}_{2}, \mathrm{Ar}, \mathrm{CO}_{2}$ exceptés); en effet $p_{I_{2}}$ $\left(-60{ }^{\circ} \mathrm{C}\right) \simeq 1,4 \times 10^{-5}$ torr tandis que $p_{\mathrm{H}_{2} \mathrm{O}}$ $\left(-60^{\circ} \mathrm{C}\right)=8 \times 10^{-3}$ torr. On maintient pendant ce temps un vide meilleur que $10^{-6}$ torr à la sortie de la 
pompe. La distillation finale se fait avec un piège refroidi à $-40^{\circ} \mathrm{C}$, en veillant tout particulièrement à n'introduire qu'une faible quantité d'iode dans la cellule car les impuretés présentes seront proportionnelles à cette quantité.

5.2 QuAlité DE CES CEllules. - La valeur de $\gamma\left(v^{\prime}=62, J^{\prime}=27\right)$ extrapolée à $p_{I_{2}}$ nulle est égale à $\gamma_{0}=(7 \pm 1) 10^{4} \mathrm{~s}^{-1}$ reproductible d'une cellule à l'autre. En trois mois, aucun vieillissement n'est décelable à cette précision de mesure.

Sur une période de deux ans de vieillissement $\gamma_{0}$ double à peu près. Cet élargissement par gaz étrangers inférieur à $10^{5} \mathrm{~s}^{-1}$ correspond donc à une pression de gaz étrangers plus de 10 fois plus petite que celle de la cellule 1 (Fig. 2) neuve.

La plupart de nos expériences ont utilisé des cellules en pyrex. Les rares essais faits sur des cellules en silice fondue ont semblé prouver qu'elles vieillissaient plus vite que les cellules en pyrex ; cependant notre expérience est trop limitée pour être totalement concluante.

Il nous semble important qu'une cellule à iode soit testée avec soin. Pour cela si l'on dispose d'un laser à Argon ionisé, l'étude de l'effet Hanle en excitation à $5017 \AA$ est une méthode très simple. Il faut seulement veiller à ne pas saturer la transition d'absorption ce qui élargirait l'effet Hanle. Une densité de puissance de l'ordre de $10^{-2} \mathrm{~W} / \mathrm{cm}^{2}$, dans le cas d'un laser multimode est parfaitement adaptée.

6. Conclusion. - Nous pensons avoir apporté dans cette note quelques éléments simples mais importants sur l'influence des phénomènes de collision régnant dans les cellules à iode. La méthode proposée pour obtenir des cellules pures, ainsi que le test suggéré, pourraient se révéler utiles au cas où la stabilisation sur l'iode du laser $\mathrm{He}-\mathrm{Ne}$ se développerait de façon systématique.

\section{Bibliographie}

[1] Hanes, G. R., Dahlstrom, C. E., Appl. Phys. Lett. 14 (1969) 362.

Hanes, G. R., Baird, K. M., Deremigis, J., Appl. Opt. 12 (1973) 1600.

SchweItzer, W. G., Jr, Kessler, E. G., Jr, Deslattes, R. D., Layer, H. P., Whetstone, J. R., Appl. Opt. 12 (1973) 2927.

Cole, J. B., Bruce, C. F., Appl. Opt. 14 (1975) 1303.

Brillet, A., Cerez, P. et Clergeot, H., I. E. E. J. Quant. Elec. 10 (1974) 526.

[2] Baxier and Grose, J. Am. Chem. Soc. 37 (1915) 1061.
[3] Shotton, K. C. and Chapman, G. D., J. Chem. Phys. 56 (1972) 1012.

[4] Broyer, M., Vigue, J. et Lehmann, J. C., J. Physique 36 (1975) 235 .

[5] Keller, J. C., Broyer, M. et Lehmann, J. C., C. R. Hebd. Séan. Acad. Sci. 277 (1973) B 369.

[6] Gouedard, G., Broyer, M., Vigue, J. and Lehmann, J. C., Chem. Phys. Lett. 43 (1976) 118.

[7] Cerez, P., Brillet, A. dans Hartmann, F., I. E. E. E. Trans. on instrumentations and measurement I. M. 23 (1974) 526.

[8] Brillet, A., Thèse d'Etat, Paris (1976) non publié. 